\title{
Dosimetric factors and Lyman normal-tissue complication modelling analysis for predicting radiation-induced lung injury in postoperative breast cancer radiotherapy: a prospective study
}

\author{
Zhi-Rui Zhou 1,2,3,*, Qing Han ${ }^{3, *}$, Shi-Xiong Liang ${ }^{3,4}$, Xiao-Dong $\mathrm{He}^{4}$, Nu-Yun $\mathrm{Cao}^{5}$ \\ and Ying-Jie $\mathbf{Z i}^{3}$ \\ ${ }^{1}$ Department of Radiation Oncology, Fudan University, Shanghai Cancer Center, Shanghai, P.R.China \\ 2 Department of Oncology, Shanghai Medical College, Fudan University, Shanghai, P.R.China \\ ${ }^{3}$ Department of Radiation Oncology, Cancer Hospital of Guangxi Medical University, Nanning, P.R.China \\ ${ }^{4}$ Department of Radiation Oncology, Shanghai Pulmonary Hospital, Tongji University School of Medicine, Shanghai, P.R.China \\ ${ }^{5}$ College of Mathematics and Information Science, Guangxi University, Nanning, P.R.China \\ * These authors have contributed equally to this paper
}

Correspondence to: Shi-Xiong Liang, email: shixiongliang@126.com

Keywords: intensity modulated radiotherapy; normal tissue complication probability; radiation-induced lung injury; breast cancer Received: September 16,2016 Accepted: October 24, $2016 \quad$ Published: October 28, 2016

Copyright: Zhou et al. This is an open-access article distributed under the terms of the Creative Commons Attribution License (CC-BY), which permits unrestricted use, distribution, and reproduction in any medium, provided the original author and source are credited.

\section{ABSTRACT}

To investigate the relationship between dosimetric factors, including Lyman normal-tissue complication (NTCP) parameters and radiation-induced lung injury (RILI), in postoperative breast cancer patients treated by intensity modulated radiotherapy (IMRT). 109 breast cancer patients who received IMRT between January 2012 and December 2013 were prospectively enrolled. A maximum likelihood analysis yielded the best estimates for Lyman NTCP parameters. Ten patients were diagnosed with RILI (primarily Grade 1 or Grade 2 RILI); the rate of RILI was $9.17 \%(10 / 109)$. Multivariate analysis demonstrated that ipsilateral lung $\mathbf{V}_{20}$ was an independent predictor $(P=0.001)$ of RILI. Setting $V_{20}=29.03 \%$ as the cut-off value, the prediction of RILI achieved high accuracy $(94.5 \%)$, with a sensitivity of $80 \%$ and specificity of $96 \%$. The NTCP model parameters for 109 patients were $m=0.437, n=0.912$, and $\operatorname{TD}_{50}(1)=17.211 \mathrm{~Gy}$. The sensitivity of the modified Lyman NTCP model to predict the RILI was $90 \%(9 / 10)$, the specificity was $69.7 \%(69 / 99)$, and the accuracy was $71.6 \%(78 / 109)$. The RILI rate of the NTCP $<9.62 \%$ in breast cancer patients was $1.43 \%(1 / 70)$, but the RILI rate of the NTCP $>9.62 \%$ in patients with breast cancer was $23.08 \%(9 / 39),(P=0.001)$. In conclusion, $V_{20}$ is an independent predictive factor for RILI in patients with breast cancer treated by IMRT; $V_{20}=\mathbf{2 9 . 0 3 \%}$ could be a useful dosimetric parameter to predict the risk of RILI. The Lyman NTCP model parameters of the new value $(m=0.437, n=0.912$, TD50 $(1)=17.211 \mathrm{~Gy})$ can be used as an effective biological index to evaluate the risk of RILI.

\section{INTRODUCTION}

Breast cancer is the most common cancer of women worldwide [1-3]. As in most other countries, the health burden of cancer is increasing in China, with more than 1.6 million people being diagnosed and 1.2 million people dying of the disease each year $[2,4,5]$. Fan Lei stated that the annual number of new cases of breast cancer and death in China accounted for $12.2 \%$ and $9.6 \%$ of cases worldwide, respectively [4]. Postoperative adjuvant radiotherapy can significantly reduce the recurrence and mortality of breast cancer and improve the quality of life for breast cancer patients [6-8].

In recent years, intensity-modulated radiation therapy (IMRT) has achieved a good curative effect in the treatment of breast cancer. In the process of breast 
cancer radiotherapy, lung tissue is affected by the dose of irradiation, resulting in radiation-induced lung injury (RILI) of different degrees. The main manifestation of RILI is acute radiation pneumonitis (RP) or chronic pulmonary interstitial fibrosis (PF) [9]. RILI is one of the common complications of radiotherapy after breast cancer surgery; it negatively affects patients' quality of life and may even lead to death in cases of severe RILI [9-11]. Therefore, it is particularly important to predict the occurrence and the degree of RILI based on the successful completion of radiotherapy plans and to prevent further pulmonary radiation injuries in early stages.

Some studies have suggested that factors including age, gender, pulmonary function, lung irradiation volume and the use of chemotherapy were associated with RILI [12]. The normal tissue complication probability (NTCP) is considered to be a good predictor of RILI factors; many scholars have studied the NTCP model of pulmonary complications after radiotherapy $[10,13]$. In our study, a comprehensive analysis of breast cancer patients was performed to assess factors predictive of RILI; the Lyman NTCP model parameters were explored to determine their predictive value.

\section{RESULTS}

\section{Baseline characteristics and clinical outcome}

From January 2012 to December 2013, a total of 109 patients met the inclusion criteria; the median followup time was 13 months (6 to 26 months). Patients were diagnosed with breast cancer at ages ranging from 29 to 73 years old; the median age at diagnosis was 45 years. The patients were divided into two groups based on whether RILI developed. The absence of RILI group included 99 cases, while the RILI group comprised 10 cases; the incidence was $9.17 \%$. There were 4 cases of acute grade 1 RILI and 4 cases of acute grade 2 RILI, which occurred within the first 6 months after radiotherapy; the symptoms resolved after treatment with antibiotics and high-dose hormones. No patients experienced grade 3, 4 or 5 acute RILI. Chronic RILI was reported in 2 cases: one in grade 2 and one in grade 3. Chronic RILI occurred more than 6 months after radiotherapy. The incidence of RILI was independent of the age of patients, operation method, and clinical stage $(P>0.05)$, while the number of chemotherapy cycles was significant $\left(\chi^{2}=5.825, P<\right.$ $0.05)$. More details are shown in Table 1.

\section{Physical parameters analysis}

The average volume of ipsilateral lung $(\mathrm{t}=-4.011$, $P<0.05)$, ipsilateral lung $\mathrm{V}_{5}(\mathrm{t}=-2.771, P<0.05), \mathrm{V}_{10}$ $(\mathrm{t}=-3.683, P<0.05), \mathrm{V}_{15}(\mathrm{t}=-4.113, P<0.05), \mathrm{V}_{20}(\mathrm{t}$ $=-4.541, P<0.05), \mathrm{V}_{25}(\mathrm{t}=-2.747, P<0.05)$ and the occurrence of RILI were analysed; the results are shown in Table 2.

\section{Multivariate logistic regression analysis and $\mathrm{ROC}$ curve}

Logistic regression analysis showed that $V_{20}$ determines the independent risk factors of RILI $(\mathrm{OR}=$ 2.618, OR value of $95 \%$ CI 1.447 to 4.737 ), Table 3 . ROC curve analysis suggested that ipsilateral lung $\mathrm{V}_{20}$ could predict RILI with an area under the ROC curve (AUC) of 0.909 . The AUC of $95 \%$ CI was 0.798 to 1.000 , and $\mathrm{V}_{20}$ of $29.03 \%$ was the best cut-off point; the sensitivity of the prediction of RILI was $80 \%$, and the specificity was $96 \%$ (Figure 1).

\section{Model parameters for Lyman NTCP}

The model parameters that were calculated in the 109 cases of breast cancer for Lyman NTCP were $\mathrm{n}=0.912, \mathrm{~m}=0.437$, and $\operatorname{TD} 50(1)=17.211$ Gy. The relationship between the mean dose and NTCP in the 109 breast cancer patients in the ipsilateral lung is shown in Figure 2.

\section{ROC curve of NTCP}

As shown in Figure 3, the ROC curve from 109 breast cancer patients had an IMRT NTCP value with AUC of $0.789,95 \%$ CI $(0.687,0.891)$, with NTCP = $9.62 \%$ as the best cut-off value; the sensitivity of the prediction model of RILI was $0.900(9 / 10)$, the specificity was 0.697 (69/99), and the accuracy was $0.716(78 / 109)$. The positive predictive value was $0.230(9 / 39)$ and the negative predictive value was $0.986(69 / 70)$. In 109 cases of breast cancer, NTCP less than $9.62 \%$ patients with breast cancer had an incidence of RILI of $1.43 \%(1 / 70)$, which is far lower than in the NTCP more than $9.62 \%$ breast cancer patients with RILI, who had an incidence of $23.08 \%(9 / 39)$; there was significant difference between two arms $(p=0.001)$, Figure 3 .

\section{DISCUSSION}

RILI includes early acute radiation pneumonia and late chronic radiation pulmonary fibrosis [9]. Clinical data suggested that the whole lung irradiation dose could not exceed $20 \mathrm{~Gy}$; the ipsilateral lung $\mathrm{V}_{20}$ less than $30 \%$ is safe [14]. Some studies suggested that the occurrence of RILI is associated with the use of chemotherapy and tamoxifen $[11,15,16]$. Some prospective studies found that lung 
Table 1: The univariate analysis of radiation-induced lung injury (RILI) and clinical factors in 109 breast cancer patients.

\begin{tabular}{|c|c|c|c|c|c|}
\hline \multicolumn{2}{|l|}{ Clinicopathology factors } & Lung injury group $(n=10)$ & No injury group $(n=99)$ & $N$ & P value \\
\hline \multirow[t]{2}{*}{ Age(years) } & $\leq 60$ & 9 & 92 & 101 & 0.735 \\
\hline & $>60$ & 1 & 7 & 8 & \\
\hline \multirow{2}{*}{$\begin{array}{l}\text { The number of cycles of } \\
\text { chemotherapy }\end{array}$} & $\leq 6$ & 2 & 64 & 66 & 0.016 \\
\hline & $>6$ & 8 & 35 & 43 & \\
\hline \multirow{2}{*}{ Operation method } & $\begin{array}{l}\text { Breast conserving } \\
\text { surgery }\end{array}$ & 5 & 56 & 61 & 0.949 \\
\hline & $\begin{array}{l}\text { Modified radical } \\
\text { mastectomy }\end{array}$ & 5 & 43 & 48 & \\
\hline \multirow{3}{*}{ Clinical staging* } & Stage I & 2 & 29 & 31 & 0.172 \\
\hline & Stage II & 2 & 41 & 43 & \\
\hline & Stage III & 6 & 29 & 35 & \\
\hline
\end{tabular}

*According to the seventh edition of AJCC staging criteria

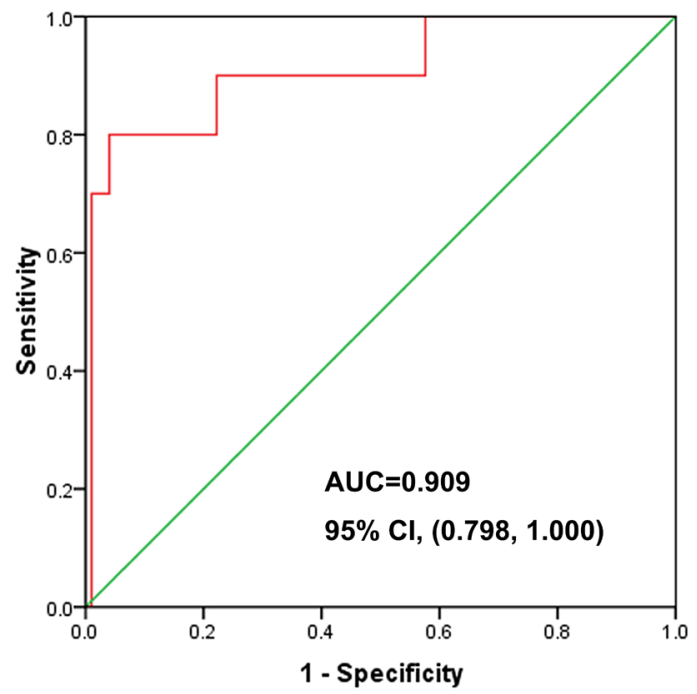

Figure 1: ROC curve analysis of $\mathbf{V}_{\mathbf{2 0}}$ AUC $=0.909,95 \% \mathrm{CI}(0.798,1.000)$.

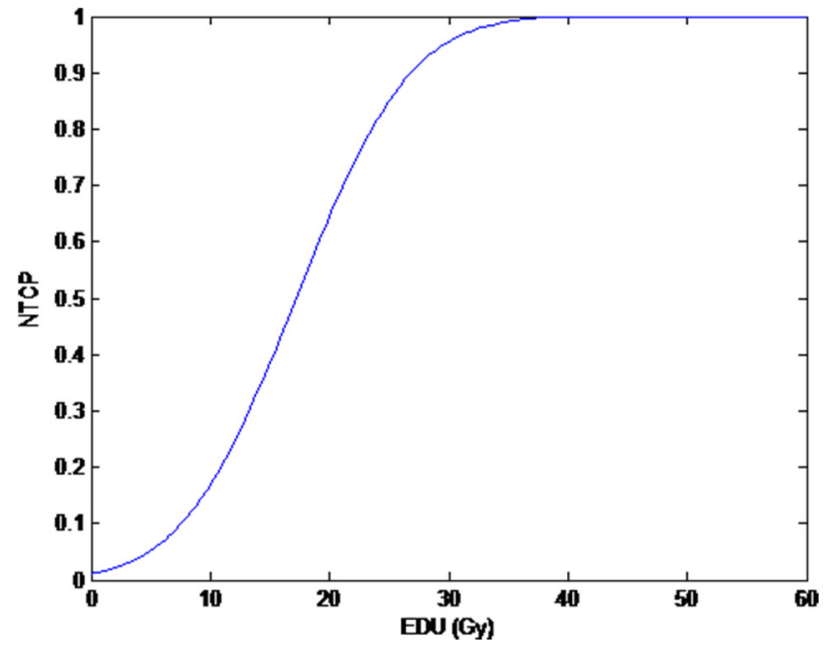

Figure 2: The relationship between NTCP and Mean dose to ipsilateral lung in 109 breast cancer cases $(n=0.912, \mathrm{~m}=$ 0.437, TD50(1) = 17.211 Gy). EUD: equivalent uniform dose. 
Table 2: The univariate analysis of radiation-induced lung injury (RILI) and physical parameters (X \pm SD) in 109 breast cancer patients.

\begin{tabular}{|l|l|l|l|}
\hline Dosmetry factors & Lung injury group $(\boldsymbol{n}=\mathbf{9 9})$ & No injury group (n=10) & P value \\
\hline $\mathrm{V}_{5}(\%)$ & $67.0 \pm 15.1$ & $80.6 \pm 10.4$ & 0.007 \\
\hline $\mathrm{V}_{10}(\%)$ & $46.51 \pm 11.7$ & $61.1 \pm 14.5$ & 0.000 \\
\hline $\mathrm{V}_{15}(\%)$ & $33.6 \pm 5.8$ & $41.9 \pm 8.4$ & 0.000 \\
\hline $\mathrm{V}_{20}(\%)$ & $25.9 \pm 2.5$ & $29.6 \pm 1.6$ & 0.000 \\
\hline $\mathrm{V}_{25}(\%)$ & $21.4 \pm 2.3$ & $23.5 \pm 2.1$ & 0.007 \\
\hline $\mathrm{V}_{30}(\%)$ & $17.9 \pm 2.3$ & $18.9 \pm 2.6$ & 0.227 \\
\hline $\mathrm{V}_{35}(\%)$ & $14.6 \pm 2.6$ & $14.5 \pm 3.0$ & 0.954 \\
\hline $\mathrm{V}_{40}(\%)$ & $11.0 \pm 2.9$ & $10.2 \pm 3.4$ & 0.355 \\
\hline $\mathrm{V}_{45}(\%)$ & $7.0 \pm 2.9$ & $5.7 \pm 3.4$ & 0.181 \\
\hline $\mathrm{PTV}\left(\mathrm{V} / \mathrm{cm}^{3}\right)$ & $882.6 \pm 335.1$ & $931.8 \pm 282.6$ & 0.655 \\
\hline Ipsilateral lung volume $\left(\mathrm{V} / \mathrm{cm}^{3}\right)$ & $1230.4 \pm 294.3$ & $1339.9 \pm 385.8$ & 0.279 \\
\hline Bilateral lung volume $\left(\mathrm{V} / \mathrm{cm}^{3}\right)$ & $2444.3 \pm 592.0$ & $2798.7 \pm 702.3$ & 0.079 \\
\hline The maximum dose of the lungs (D/Gy) & $55.7 \pm 3.0$ & $55.7 \pm 2.5$ & 0.949 \\
\hline The average dose of bilateral lung (D/Gy) & $8.6 \pm 1.5$ & $9.4 \pm 1.1$ & 0.101 \\
\hline The maximum dose of contralateral lung (D/Gy) & $16.8 \pm 14.9$ & $18.5 \pm 13.6$ & 0.726 \\
\hline The average dose of contralateral lung (D/Gy) & $1.8 \pm 2.2$ & $1.8 \pm 0.9$ & 0.922 \\
\hline The maximum dose of ipsilateral lung (D/Gy) & $55.6 \pm 2.9$ & $55.5 \pm 2.3$ & 0.928 \\
\hline The average dose of ipsilateral lung (D/Gy) & $14.8 \pm 1.6$ & 0.000 \\
\hline & & & \\
\hline
\end{tabular}

injury is associated with age and individual radiation susceptibility $[12,17]$. Other studies have reported that smoking may reduce the incidence of RILI [18]. Our study focuses on the dosimetric parameters and Lyman NTCP model related to RILI to predict the incidence of RILI, optimize the radiotherapy plan and improve the patients' quality of life. The study group patients did not have smoking history and took no endocrine drugs during the radiotherapy period. The study results show that age has no effect on the occurrence of RILI $(P>0.05)$. Considering the effect of a small number of cases or short follow-up time, the clinical staging and operation effect on RILI manifested no statistical significance.

The lung $\mathrm{V}_{20}$, mean dose of normal lung (MLD) and other dosimetric parameters are predictive of RILI. When MLD $\leq 17 \mathrm{~Gy}, \mathrm{~V}_{20} \leq 31.0 \%$, and $\mathrm{V}_{30} \leq 24.0 \%$, the probability of severe RILI will be $0-10.7 \%[19,20]$. Claude et al. [21] reported that for MLD, $\mathrm{V}_{20}$ for level 1 and level 1 above a meaningful forecast, RILI, MLD and $\mathrm{V}_{20}$ are associated with grade 2 or greater RILI. Graham et al. [22] found that single factor analysis suggests that the discretion of the incidence of RILI and severity are closely associated with irradiated lung volume and dose. Multivariable analysis found that $\mathrm{V}_{20}$ is the most important factor that affects RILI; when $\mathrm{V}_{20}<22 \%$, no RILI occurs, when $\mathrm{V}_{20}$ is between $22 \%$ and $31 \%, 32 \%$ and $40 \%$ and $>40 \%$, the 24 month RILI incidence is $7 \%, 13 \%$ and $7 \%$, respectively. At the same time, when $\mathrm{V}_{20}$ is between $22 \%$ and $31 \%, 8 \%$ of patients have 2nd level RILI, while 3rd level radioactive pneumonia occurs when $V_{20} \geq 32 \%$. Asakura et al. [23] found that $\mathrm{V}_{20}$ is an independent risk factor in patients with oesophageal synchronous chemotherapy; when $\mathrm{V}_{20}$ $<24 \%$, the incidence of RILI is $13 \%$, when $25 \%<\mathrm{V}_{20}<$ $36 \%$, the rate is $33 \%$ and when $V_{20} \geq 37 \%$, the incidence is $78 \%$. Additionally, some scholars demonstrated that in normal lungs, MLD and the occurrence of RILI also showed a positive correlation; here, with the increase of MLD, the incidence of RILI also gradually increased. 
Table 3: Odds ratio and 95\% CI for RILI from multivariate analysis of 109 breast cancer patients

\begin{tabular}{|l|c|c|c|}
\hline \multicolumn{1}{|c|}{ Factors } & Pvalue & OR & 95\%CI \\
\hline V5 $(\%)$ & 0.471 & 1.051 & $0.919-1.202$ \\
\hline V10 $(\%)$ & 0.531 & 1.060 & $0.883-1.273$ \\
\hline V15(\%) & 0.965 & 1.006 & $0.784-1.289$ \\
\hline V20 $(\%)$ & 0.001 & 2.618 & $1.447-4.737$ \\
\hline V25(\%) & 0.995 & 1.004 & $0.318-3.172$ \\
\hline The average dose of ipsilateral lung (Gy) & 0.462 & 0.628 & $0.181-2.173$ \\
\hline The number of cycles of chemotherapy & 0.076 & 8.488 & $0.798-90.317$ \\
\hline
\end{tabular}

Roeder et al. [24] found that when the MLD $<10 \mathrm{~Gy}$, the incidence of RILI was 7\%; when 10 Gy $<$ MLD $<$ $20 \mathrm{~Gy}$, the incidence of RILI was 19\%; and when MLD $>20 \mathrm{~Gy}$, the incidence of RILI increased significantly. Therefore, V20 and Dmean should be chosen to measure the parameters of RILI. Univariate factor analysis shows that the RILI in chemotherapy cycle number, the average dose of lung and $\mathrm{V}_{5}-\mathrm{V}_{25}$ had obvious differences $(p<$ $0.05)$; the logistic regression analysis showed that $\mathrm{V}_{20}$ had the closest relationship with RILI. When $\mathrm{V}_{20}>29.03 \%$, the incidence of RILI was $66.67 \%$, and $\mathrm{V}_{20}$ acuities were $29.03 \%$ when the incidence of RILI was $2.06 \%$; the difference between the two was statistically significant $(p=0.000)$. The results suggest that $\mathrm{V}_{20}$ can serve as a predictor of RILI occurrence and evaluate the merits of the radiotherapy plans. Therefore, according to $\mathrm{V}_{20} \leq 29.03 \%$, the parameter values for predicting the occurrence of RILI can effectively reduce the incidence of RILI and improve the quality of life in breast cancer patients.

The lung injury induced by chemotherapy drugs and RILI can produce superimposed effects [25]. Theuws et al. found that patients with breast cancer receiving chemotherapy plus radiotherapy in the application of CMF showed more of a decline in lung function than with simple radiotherapy, with a significant result [26]. Dang et al. analysed 93 RILI patients with non-small cell lung cancer; in combined radiation and chemotherapy patients, the incidence of RILI was $61.1 \%$, which was significantly higher than the $42.1 \%$ reported in radiation therapy alone [27]. Our data analysis showed that the incidence of lung injury in the chemotherapy group undergoing $>6$ cycles is higher than in the group receiving 6 or fewer chemotherapy cycles $(p<0.05)$. This study does not provide a detailed analysis of the impact of different chemotherapy regimens on RILI because the selected cases were included for various reasons (including an

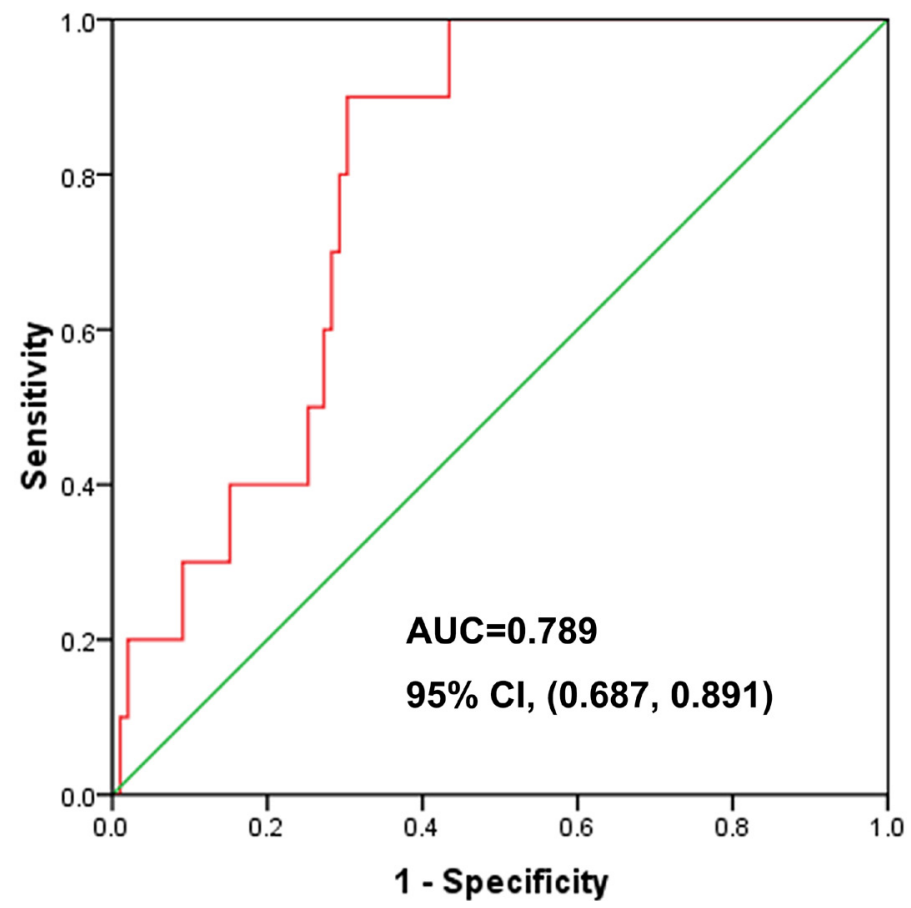

Figure 3: One hundred and nine cases of postoperative breast cancer patients after IMRT; ROC curve of NTCP, each of which had a projected NTCP value using the NTCP model $\left(n=0.912, \mathrm{~m}=0.437, \mathrm{TD}_{50}(1)=17.211 \mathrm{~Gy}\right), \mathrm{AUC}=0.789$, 95\% CI (0.687, 0.891). 
inability to tolerate side effects during the chemotherapy period, and personal economic reasons meaning that the patient could not afford to complete chemotherapy cycles). However, chemotherapy failed to enter the multivariable analysis. The logistic regression model is the main reason that the research analysis may have not considered the interactions between all factors, ignoring the influence between them.

The Lyman NTCP model is derived from the DVH graph for mathematics-related data to calculate the probability of complications after radiotherapy in patients. In 1991, Burman found the Emani data value fitting model parameters $\mathrm{n}, \mathrm{m}$, and $\mathrm{TD}_{50}$ of the lung values to be $0.87,0.18$, and $24.5 \mathrm{~Gy}$, which have a large volume effect on RILI [28]; this also means that NTCP relies more on the whole volume according to the average dose. For the study of RILI, Lyman NTCP model parameters showed some differences in the patient population and disease; as a result, the calculated parameters are not the same, but generally reflect the effect of lung volume, and $n$ value is 0.87 to 1.0 . Shi et al. analysed 94 patients with locally advanced non-small cell lung cancer [29]; for the resulting NTCP multiple factors, regression analysis found that NTCP was $4.2 \%$, which was used as a cut-off value point. When NTCP acuities were $4.2 \%$ and $>4.2 \%$, the incidence of RILI was $1.4 \%$ and $43.5 \%$, respectively. Using maximum likelihood method fitting, this study collected the new parameter of the model as $n=0.912, \mathrm{~m}=0.437$, $\mathrm{TD}_{50}(1)=17.211 \mathrm{~Gy}$, and $\mathrm{NTCP}=9.62 \%$ as the diagnostic values; the model to predict the sensitivity of RILI was $0.9(9 / 10)$, with a specific degree of 0.697(69/99), and an accuracy of 0.716 (78/109). In 109 breast cancer patients, NTCP $<9.62 \%$ of patients showed an incidence of RILI of $1.43 \%(1 / 70)$, which was far lower than the NTCP $\geq$ $9.62 \%$ of breast cancer patients, who had a RILI incidence rate of $23.08 \%(9 / 39)$, suggesting that the model had a good prediction effect on RILI. Tsougos analysed the NTCP model and found its predictive value in non-small cell lung cancer patients with RILI [30]; if the calculation of the ipsilateral lung NTCP, and the clinical results of NTCP model on the value of the (RTOG grade 2 RILI) are in good agreement, and the left and right side of the lung as a whole to calculate the value of NTCP is calculated, values of the model are not very good with regard to lung injury by clinical observation, and the clinical incidences underestimated. All of the cases were collected along with dosimetry data for ipsilateral breast tumour of the lung; it did not compare the contralateral and bilateral pulmonary NTCP values.

In conclusion, NTCP could be used for the evaluation of the radiotherapy target in the optimization of IMRT. The Lyman NTCP model parameters of the new value $\left(\mathrm{m}=0.437, n=0.912, \mathrm{TD}_{50}(1)=17.211 \mathrm{~Gy}\right)$ can be used as an effective index to evaluate the risk of occurrence of RILI. In addition, $\mathrm{V}_{20}$ was an independent predictive factor for RILI in patients with breast cancer treated by IMRT. $\mathrm{V}_{20}=29.03 \%$ could be a useful dosimetric parameter for evaluating the risk of RILI.

\section{MATERIALS AND METHODS}

\section{Patients and inclusion criteria}

The study prospectively included all of the female breast cancer patients who were treated after surgery in the Cancer Hospital of Guangxi Medical University between January 2012 and December 2013. Inclusion criteria: (1) female patients who underwent breast cancer surgery in the hospital with breast cancer confirmed by postoperative pathology diagnosis; (2) age from 25 to 75 years old; (3) Karnofsky score (Karnofsky performance status, KPS) $\geq 70$ points; (4) no smoking history; (5) patients who underwent IMRT for the first time; (6) no concurrent chemotherapy and endocrine therapy during radiotherapy; and (7) patients completed radiotherapy and were followed-up for more than 6 months. Exclusion criteria: (1) patients with tumour recurrence or distant metastasis; (2) patients with concurrent heart or lung disease; (3) patients who could not tolerate radiotherapy or failed to complete radiation therapy for any reason. This study was approved by the Institutional Review Board of Cancer Hospital of Guangxi Medical University, which required informed consent.

\section{Treatment methods}

IMRT was performed using a vacuum pad in a fixed position. The CT scan imaging system was networked with the radiotherapy planning system, delineating clinical target volume (CTV) and organ at risk (OAR), including spinal cord, heart, lung and contralateral breast. The planning target volume (PTV) is an extension of CTV. The dose of radiotherapy after breast-conserving surgery was as follows: whole breast irradiation with $6 \mathrm{MV}$ X-rays, with a total dose of $50 \mathrm{~Gy} / 5 \mathrm{~W}\left(2 \mathrm{~Gy} / \mathrm{f} \bullet \mathrm{d}^{-1}, 5 \mathrm{f} / \mathrm{W}\right)$. The supraclavicular region was used, and exposure of the whole breast was prevented with axillary equipment. The breast tumour bed synchronous dosage was 10-16 $\mathrm{Gy} / 1-1.5 \mathrm{~W}\left(2 \mathrm{~Gy} / \mathrm{f}^{\circ} \mathrm{d}^{-1}, 5 \mathrm{f} / \mathrm{W}\right)$ in patients with invasive breast cancer and negative margins; the breast tumour bed boost was $16-20 \mathrm{~Gy} / 1.5-2 \mathrm{~W}\left(2 \mathrm{~Gy} / \mathrm{f}^{\circ} \mathrm{d}^{-1}, 5 \mathrm{f} / \mathrm{W}\right)$ in patients with invasive breast cancer and positive margins. After modified radical mastectomy for breast cancer, the radiotherapy dose was as follows: using $6 \mathrm{MV} \mathrm{X}$-ray irradiation $\left(2 \mathrm{~Gy} / \mathrm{f}, 50 \mathrm{~Gy} / 5 \mathrm{~W} \cdot \mathrm{d}^{-1}, 5 \mathrm{f} / \mathrm{W}\right)$. All patients who underwent modified radical mastectomy had treatment of the chest wall surface using a $0.5 \mathrm{~cm}$ thick film pad when irradiated equivalent to $20 \mathrm{~Gy}$, and then the equivalent film was removed when irradiated to $50 \mathrm{~Gy}$. The treatment plan required that $95 \%$ of the target dose covered the target 
volume of $100 \%$, and the maximum dose was less than $110 \%$ of the prescribed dose. The PTV dose and organ damage limits were: ipsilateral lung, $\mathrm{V}_{20}<30 \%, \mathrm{D}_{\text {mean }}<20$ Gy; bilateral lung, $\mathrm{V}_{20}<20 \%$; heart (only left-sided breast cancer patients), $\mathrm{V}_{30}<10 \%, \mathrm{~V}_{40}<5 \%$; and contralateral breast, $\mathrm{D}_{\text {mean }}<1 \mathrm{~Gy}, \mathrm{D}_{\max }<5$ Gy.

\section{Diagnosis and evaluation of RILI}

According to the American Radiation Therapy Oncology Group (radiation therapy, oncology group, RTOG), acute and chronic RILI can be divided into 5 levels based on standard RILI evaluation [31]. After radiotherapy, following up for 1 to 6 months and for more than 6 months was used to diagnose acute radiation pneumonia and chronic RILI.

\section{Clinical and physical parameters}

RILI-related clinical factors such as age, clinical operation, tumour stage, number of chemotherapy cycles and physical parameters, including the planning target volume (PTV), ipsilateral lung and bilateral lungs volume, bilateral lungs, ipsilateral lung and contralateral lung maximum dose, mean dose, and lung $\mathrm{V}_{5}, \mathrm{~V}_{10}, \mathrm{~V}_{15}$, $\mathrm{V}_{20}, \mathrm{~V}_{25}, \mathrm{~V}_{30}, \mathrm{~V}_{35}, \mathrm{~V}_{40}$, and $\mathrm{V}_{45}$ (i.e., the ipsilateral lung received $5,10,15,20,25,30,35,40$, and 45 Gy dose of the lung volume to the total lung volume percentage) were prospectively collected.

\section{The model of Lyman NTCP}

The Dose Volume Histogram (DVH) data for each patient were transferred to MATLAB (version R2009b, MathWorks, USA) software. The data were fitted using the Lyman-Kutcher-Burman (LKB) NTCP model [10, 13, 32]. The Lyman NTCP model formula is as follows [32]:

$$
\mathrm{NTCP}=\phi(t)=\frac{1}{\sqrt{2 \pi}} \int_{-\infty}^{t} e^{-\frac{x^{2}}{2}} d x, t=\left(\frac{D-T D_{50}(V)}{m \cdot T D_{50}(V)}\right), T D_{50}(v)=T D_{50}(1) \cdot v^{-n}
$$

The Lyman NTCP model only considers uniform illumination; the radiation dose of normal lung radiotherapy in breast cancer is not uniform, with KutcherBurman equivalent volume calculation method used to normalize non-uniform dose-distribution (DVH plots) converted into equivalent uniform distribution ${ }^{[10]}$. An equivalent volume is defined as the volume of irradiated lung tissue that receives a considerable probability of lung injury due to uniform illumination and the real situation of uneven irradiation. The most widely used NTCP model for radiation pneumonitis (RP) is the LKB model. This model has three parameters, a position parameter, $\mathrm{TD}_{50}$, a steepness parameter, $m$, and the volume exponent, $n$ (where $n=1$ the model reverts to mean lung dose; MLD). While $\mathrm{TD}_{50}$ is strongly dependent on the grade of RP being considered, $\mathrm{n}$ is often regarded as a tissue characteristic. The Newton-Raphson method was used to calculate $\mathrm{m}$, $\mathrm{n}, \mathrm{TD}_{50}$ and maximum likelihood parameter estimation by MATLAB [10, 13, 32].

\section{Statistical analysis}

SPSS22.0 (IBM SPSS, NY, USA) statistical software was used for analysis. Univariate analysis using the $\mathrm{t}$ test and $\chi^{2}$ test and multivariate analysis using the logistic regression model. Receiver operating characteristic (ROC) curve analysis of ipsilateral lung volume percentage was in relation to the RILI. In each case, the NTCP value with the Lyman-Kutcher-Burman model was calculated; the relationship between the parameters and the occurrence of RILI was analysed using the $t$ test, and the difference between the two groups in the model parameters was compared using the t test. Receiver operating characteristic (ROC) curve analysis of the new Lyman model was performed to calculate the relationship between the NTCP value and RILI. $P<0.05$ was considered statistically significant.

\section{CONFLICTS OF INTEREST}

The authors declare no conflicts of interest.

\section{Author contributions}

Conception and design: Shi-Xiong Liang. Collection and assembly of data: Zhi-Rui Zhou, Qing Han and ShiXiong Liang. Data analysis and interpretation: Qing Han, Zhi-Rui Zhou and Shi-Xiong Liang. Manuscript writing: All authors. Final approval of manuscript: All authors. Financial support: Shi-Xiong Liang.

\section{REFERENCES}

1. Siegel RL, Miller KD and Jemal A. Cancer statistics, 2016. CA Cancer J Clin. 2016; 66:7-30.

2. Levaggi A, Poggio F and Lambertini M. The burden of breast cancer from China to Italy. Journal of Thoracic Disease. 2014; 6:591-594.

3. Jia M, Zheng R, Zhang S, Zeng H, Zou X and Chen W. Female breast cancer incidence and mortality in 2011, China. Journal of Thoracic Disease. 2015; 7:1221-1226.

4. Fan L, Strasser-Weippl K, Li JJ, St Louis J, Finkelstein DM, Yu KD, Chen WQ, Shao ZM and Goss PE. Breast cancer in China. Lancet Oncol. 2014; 15:e279-289.

5. Xu B, Hu X, Jiang Z, Li H, Chen J, Cui S, Li Q, Liao N, Liu D, Liu J, Lu J, Shen K, Sun T, et al. National consensus in China on diagnosis and treatment of patients with advanced 
breast cancer. Translational Cancer Research. 2015; 4:557573.

6. Clarke M, Collins R, Darby S, Davies C, Elphinstone P, Evans V, Godwin J, Gray R, Hicks C, James S, MacKinnon E, McGale P, McHugh T, et al. Effects of radiotherapy and of differences in the extent of surgery for early breast cancer on local recurrence and 15-year survival: an overview of the randomised trials. Lancet. 2005; 366:2087-2106.

7. Overgaard M, Hansen PS, Overgaard J, Rose C, Andersson M, Bach F, Kjaer M, Gadeberg CC, Mouridsen HT, Jensen $\mathrm{MB}$ and Zedeler K. Postoperative radiotherapy in high-risk premenopausal women with breast cancer who receive adjuvant chemotherapy. Danish Breast Cancer Cooperative Group 82b Trial. N Engl J Med. 1997; 337:949-955.

8. Overgaard M, Jensen MB, Overgaard J, Hansen PS, Rose C, Andersson M, Kamby C, Kjaer M, Gadeberg CC, Rasmussen BB, Blichert-Toft M and Mouridsen HT. Postoperative radiotherapy in high-risk postmenopausal breast-cancer patients given adjuvant tamoxifen: Danish Breast Cancer Cooperative Group DBCG 82c randomised trial. Lancet. 1999; 353:1641-1648.

9. Blom Goldman U, Wennberg B, Svane G, Bylund H and Lind P. Reduction of radiation pneumonitis by V20constraints in breast cancer. Radiat Oncol. 2010; 5:99.

10. Gagliardi G, Bjohle J, Lax I, Ottolenghi A, Eriksson F, Liedberg A, Lind P and Rutqvist LE. Radiation pneumonitis after breast cancer irradiation: analysis of the complication probability using the relative seriality model. Int J Radiat Oncol Biol Phys. 2000; 46:373-381.

11. Taghian AG, Assaad SI, Niemierko A, Kuter I, Younger J, Schoenthaler R, Roche M and Powell SN. Risk of pneumonitis in breast cancer patients treated with radiation therapy and combination chemotherapy with paclitaxel. J Natl Cancer Inst. 2001; 93:1806-1811.

12. Rancati T, Ceresoli GL, Gagliardi G, Schipani S and Cattaneo GM. Factors predicting radiation pneumonitis in lung cancer patients: a retrospective study. Radiother Oncol. 2003; 67:275-283.

13. Seppenwoolde Y, Lebesque JV, de Jaeger K, Belderbos JS, Boersma LJ, Schilstra C, Henning GT, Hayman JA, Martel MK and Ten Haken RK. Comparing different NTCP models that predict the incidence of radiation pneumonitis. Normal tissue complication probability. Int J Radiat Oncol Biol Phys. 2003; 55:724-735.

14. Lind PA, Svane G, Gagliardi G and Svensson C. Abnormalities by pulmonary regions studied with computer tomography following local or local-regional radiotherapy for breast cancer. Int J Radiat Oncol Biol Phys. 1999; 43:489-496.

15. Bentzen SM, Skoczylas JZ, Overgaard M and Overgaard J. Radiotherapy-related lung fibrosis enhanced by tamoxifen. J Natl Cancer Inst. 1996; 88:918-922.

16. Lind PA, Marks LB, Jamieson TA, Carter DL, Vredenburgh JJ, Folz RJ and Prosnitz LR. Predictors for pneumonitis during locoregional radiotherapy in high-risk patients with breast carcinoma treated with high-dose chemotherapy and stem-cell rescue. Cancer. 2002; 94:2821-2829.

17. Lind PA, Bylund H, Wennberg B, Svensson C and Svane $\mathrm{G}$. Abnormalities on chest radiographs following radiation therapy for breast cancer. Eur Radiol. 2000; 10:484-489.

18. Bjermer L, Franzen L, Littbrand B, Nilsson K, Angstrom $\mathrm{T}$ and Henriksson R. Effects of smoking and irradiated volume on inflammatory response in the lung of irradiated breast cancer patients evaluated with bronchoalveolar lavage. Cancer Res. 1990; 50:2027-2030.

19. Kim TH, Cho KH, Pyo HR, Lee JS, Zo JI, Lee DH, Lee JM, Kim HY, Hwangbo B, Park SY, Kim JY, Shin KH and Kim DY. Dose-volumetric parameters for predicting severe radiation pneumonitis after three-dimensional conformal radiation therapy for lung cancer. Radiology. 2005; 235:208-215.

20. Chang DT, Olivier KR, Morris CG, Liu C, Dempsey JF, Benda RK and Palta JR. The impact of heterogeneity correction on dosimetric parameters that predict for radiation pneumonitis. Int J Radiat Oncol Biol Phys. 2006; 65:125-131.

21. Claude L, Perol D, Ginestet C, Falchero L, Arpin D, Vincent M, Martel I, Hominal S, Cordier JF and Carrie C. A prospective study on radiation pneumonitis following conformal radiation therapy in non-small-cell lung cancer: clinical and dosimetric factors analysis. Radiother Oncol. 2004; 71:175-181.

22. Graham MV, Purdy JA, Emami B, Harms W, Bosch W, Lockett MA and Perez CA. Clinical dose-volume histogram analysis for pneumonitis after 3D treatment for non-small cell lung cancer (NSCLC). Int J Radiat Oncol Biol Phys. 1999; 45:323-329.

23. Asakura H, Hashimoto T, Zenda S, Harada H, Hirakawa K, Mizumoto M, Furutani K, Hironaka S, Fuji H, Murayama $\mathrm{S}$, Boku $\mathrm{N}$ and Nishimura T. Analysis of dose-volume histogram parameters for radiation pneumonitis after definitive concurrent chemoradiotherapy for esophageal cancer. Radiother Oncol. 2010; 95:240-244.

24. Roeder F, Friedrich J, Timke C, Kappes J, Huber P, Krempien R, Debus J and Bischof M. Correlation of patientrelated factors and dose-volume histogram parameters with the onset of radiation pneumonitis in patients with small cell lung cancer. Strahlenther Onkol. 2010; 186:149-156.

25. Robnett TJ, Machtay M, Vines EF, McKenna MG, Algazy KM and McKenna WG. Factors predicting severe radiation pneumonitis in patients receiving definitive chemoradiation for lung cancer. Int J Radiat Oncol Biol Phys. 2000; 48:8994.

26. Theuws JC, Kwa SL, Wagenaar AC, Boersma LJ, Damen EM, Muller SH, Baas P and Lebesque JV. Dose-effect relations for early local pulmonary injury after irradiation for malignant lymphoma and breast cancer. Radiother Oncol. 1998; 48:33-43. 
27. Dang J, Li G, Lu X, Yao L, Zhang S and Yu Z. Analysis of related factors associated with radiation pneumonitis in patients with locally advanced non-small-cell lung cancer treated with three-dimensional conformal radiotherapy. J Cancer Res Clin Oncol. 2010; 136:1169-1178.

28. Burman C, Kutcher GJ, Emami B and Goitein M. Fitting of normal tissue tolerance data to an analytic function. Int $\mathrm{J}$ Radiat Oncol Biol Phys. 1991; 21:123-135.

29. Shi A, Zhu G, Wu H, Yu R, Li F and Xu B. Analysis of clinical and dosimetric factors associated with severe acute radiation pneumonitis in patients with locally advanced nonsmall cell lung cancer treated with concurrent chemotherapy and intensity-modulated radiotherapy. Radiat Oncol. 2010; $5: 35$.
30. Tsougos I, Nilsson P, Theodorou K, Kjellen E, Ewers SB, Jarlman O, Lind BK, Kappas C and Mavroidis P. NTCP modelling and pulmonary function tests evaluation for the prediction of radiation induced pneumonitis in nonsmall-cell lung cancer radiotherapy. Phys Med Biol. 2007; 52:1055-1073.

31. Cox JD, Stetz J and Pajak TF. Toxicity criteria of the Radiation Therapy Oncology Group (RTOG) and the European Organization for Research and Treatment of Cancer (EORTC). Int J Radiat Oncol Biol Phys. 1995; 31:1341-1346.

32. Semenenko VA and Li XA. Lyman-Kutcher-Burman NTCP model parameters for radiation pneumonitis and xerostomia based on combined analysis of published clinical data. Phys Med Biol. 2008; 53:737-755. 